\title{
Airway Management in an Adult Patient With a Large Vallecular Cyst
}

\author{
Azize Bestas $^{\mathrm{a}, \mathrm{c}}$, Ismail Demirel ${ }^{\mathrm{a}}$, Irfan Kaygusuz ${ }^{\mathrm{b}}$
}

\begin{abstract}
Vallecular cysts are rarely seen in adults and occur as a result of the tongue base mucus retention. In cases with laryngeal inlet obstruction due to a vallecular cyst, the airway management sometimes might be a challenge. We presented the airway management of an adult patient with difficult airway due to a large vallecular cyst. A 28-year-old male patient with a large vallecular cyst was scheduled for surgical excision of the cyst under general anesthesia. Despite the use of different laryngoscopic blades, no laryngeal structure was seen due to the cystic formation. After aspiration of approximately $20 \mathrm{~mL}$ cyst content, the peripheral side of the epiglottis was seen on the left-upper side of the shrunken cyst. Therefore, laryngoscopy was performed by an insertion of the left side of the mouth, and tracheal intubation was achieved by endotracheal tube which was advanced blindly under the epiglottis. A week later, due to cyst reoccurrence, the patient was undergone surgery again. No problem arose during the course of anesthesia, surgical intervention and the postoperative period, and the patient was discharged on the fifth postoperative day. We concluded that the view of the glottis or other laryngeal structures can be obtained by shrinking the vallecular cyst size.
\end{abstract}

Keywords: Vallecular cyst; Airway management; Adult patient

\section{Introduction}

Vallecular cysts, which are generally known as epiglottic mucus retention or tongue base cysts, are formations that are

\footnotetext{
Manuscript accepted for publication January 28, 2014

${ }^{a}$ Department of Anesthesiology and Reanimation, Firat University School of Medicine, 23119 Elazig, Turkey

${ }^{\mathrm{b}}$ Department of Otorhinolaryngology, Firat University School of Medicine, 23119 Elazig, Turkey

${ }^{\mathrm{c} C}$ Corresponding author: Azize Bestas, Department of Anesthesiology and Reanimation, Firat University Hospital, 23119 Elazig, Turkey. Email: abestas@firat.edu.tr
}

doi: http://dx.doi.org/10.14740/jmc1970e generally seen in infants and children. They are rarely seen in adults and occur as a result of the tongue base mucus retention $[1,2]$. These cysts might be asymptomatic or might be associated with airway obstruction, feeding difficulties and failure to thrive during infancy, and even with death due to severe laryngeal obstruction [3-5]. In cases with laryngeal inlet obstruction due to a vallecular cyst, the airway management sometimes might be a challenge [5-7]. In this case report, the airway management of an adult patient with difficult airway due to a large vallecular cyst completely obstructing the pharynx was presented.

\section{Case Report}

This case report was presented after obtaining the patient's written informed consent. A 28-year-old male patient who was experiencing foreign body sensation in his throat, dysphagia, dyspnea, hoarseness and snoring that persisted for 2 months, presented with his complaints to the Otorhinolaryngology Clinic. In the indirect laryngoscopic examination, a cystic mass with a smooth surface filling vallecula was detected, and under general anesthesia, the excision of the cystic formation was scheduled. No abnormality was found during the preanesthetic routine systemic physical examination and laboratory investigations. The view of oropharyngeal structures was evaluated as Mallampati class 1. The patient was taken to the operating room without premedication and standard monitoring was applied. As patients with vallecular cyst have the potential for a difficult airway, in addition to tracheostomy and other difficult airway equipment, an otorhinolaryngology specialist was made available during anesthesia induction and tracheal intubation. Anesthesia was induced with sevoflurane in $50 \% \mathrm{O}_{2}$ and air and when necessary with bolus propofol administration. Following tracheal intubation, anesthesia was maintained with end-tidal (ET) $2.5 \%$ sevoflurane in $50 \% \mathrm{O}_{2}$-air and i.v. $1 \mu \mathrm{g} /$ $\mathrm{kg}$ fentanyl. Since mask ventilation could only be provided with two practitioners, the patient's spontaneous breathing was maintained. Direct laryngoscopic attempts and intubation were performed without muscle relaxant. Despite the use of different laryngoscopic blades (No. $=3$, Macintosh 


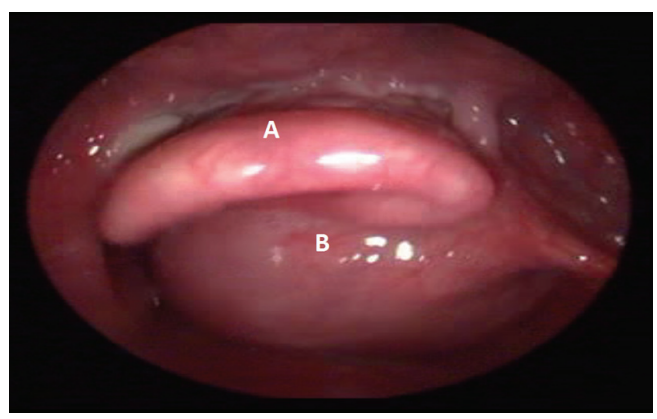

Figure 1. After aspiration of cyst content laryngoscopic view. (A) epiglottis. (B) cyst.

and Miller), no laryngeal structure was seen due to the cystic formation. Therefore, it was decided to aspirate cyst content under laryngoscopy. After aspiration of approximately $20 \mathrm{~mL}$ cyst content with a needle, the peripheral side of the epiglottis was seen on the left-upper side of the shrunken cyst (Fig. 1). Therefore, laryngoscopy was performed by an insertion of the left side of the mouth, and tracheal intubation was achieved by endotracheal tube which was advanced blindly under the epiglottis. After confirming the tracheal placement of the tube with pulmonary auscultation, $\mathrm{SpO}_{2}$ and ET $\mathrm{CO}_{2}$ monitoring, surgery was started, and surgical excision of the cyst was performed. The patient's oxygenation was maintained at normal values with mask ventilation performed with two applicators until endotracheal intubation was achieved. Eight milligrams dexamethasone was administered intravenously as a precaution against potential airway edema. Upon completing the operation, anesthesia application was finalized and tracheal extubation was performed without any problem. Histopathological examination of the excised material confirmed a vallecular cyst. A week later, due to cyst reoccurrence, the patient was operated on again. With the presence of otorhinolaryngology, difficult airway equipment was made available and anesthesia was induced with sevoflurane in $50 \%$ oxygen and air. Probably due to the fact that the size of the cyst was smaller than the previous one, neuromuscular blocking agent vecuronium was given in order to facilitate laryngoscopy and tracheal intubation since mask ventilation was easily provided. Upon the request of the surgeon, cyst content was not aspirated. The previous experience was taken into account, direct laryngoscopy was performed through insertion of the left side of the mouth and tracheal intubation was achieved with a single attempt. No problem arose during the course of anesthesia, surgical intervention and the postoperative period, and the patient was discharged on the fifth postoperative day.

\section{Discussion}

The size of a vallecular cyst has a tendency to increase with mucus secretion. As in our patient, since the large cyst prevented the view of the larynx, the airway management is of particular importance. In the cases where the larynx inlet is completely closed by the cystic formation, generally more than one intervention is performed and tracheal intubation can be performed by using laryngoscopes designed for difficult airway, fiberoptic or rigid bronchoscopy [6-8]. Unfortunately, we have not a fiberoptic bronchoscopy or a video laryngoscope device. In some cases, mask ventilation is achieved with great difficulty, direct laryngoscopic oral intubation might not be achieved and urgent tracheostomy might be required $[5,8]$. In the cases in which laryngeal view is blocked by the cyst, glottis might be visualized with the aspiration of the cyst content and tracheal intubation might be achieved. Vijayanand et al [9] reported that in an adult patient with a large $(4 \times 3 \mathrm{~cm}$ size $)$ vallecular cyst occupying all of the tongue base, epiglottis and larynx are apparent upon the aspiration of the cyst content with a needle under local anesthesia, and endotracheal intubation could be easily performed on the next day under general anesthesia. Probably we were able to see a part of the epiglottis since we could not completely aspirate the cyst content.

Computed tomography and magnetic resonance imaging examinations showed that the position of the epiglottis can be changed according to the placement of the cyst [10]. In our case, after draining a large portion of cyst content, we detected that the epiglottis was pushed to left side by the vallecular cyst located in the right side of the tongue base. Therefore, although direct laryngoscopy is performed through insertion of the right side of the mouth, we performed direct laryngoscopy and endotracheal intubation through the insertion of the left side of the mouth. Cheng et al [10] revealed that the laryngeal inlet was obstructed by the cyst in a 50-day-old infant with a large vallecular cyst, when the blade was on the midline. In a similar manner we performed, they moved the blade to $45^{\circ}$ left from the midline, and they placed it laterally and achieved intubation.

As a result, they are able to obtain the view of the glottis or other laryngeal structures by shrinking the vallecular cyst size by needle aspiration of its content. Despite the fact that routine direct laryngoscopy is performed by entering from the right side of the mouth, performing laryngoscopy through insertion of the midline or left side, alternatively, can provide laryngeal view and facilitate tracheal intubation.

\section{Grant Support}

None.

\section{References}

1. Prowse S, Knight L. Congenital cysts of the infant lar- 
ynx. Int J Pediatr Otorhinolaryngol. 2012;76(5):708711.

2. Chen EY, Lim J, Boss EF, Inglis AF, Jr., Ou H, Sie KC, Manning SC, et al. Transoral approach for direct and complete excision of vallecular cysts in children. Int $\mathbf{J}$ Pediatr Otorhinolaryngol. 2011;75(9):1147-1151.

3. Jahagirdar SM, Karthikeyan P, Ravishankar M. Acute airway obstruction, an unusual presentation of vallecular cyst. Indian J Anaesth. 2011;55(5):524-527.

4. Gilbey P, Amirav I, Luder A. Vallecular cyst: rare cause of failure to thrive without respiratory distress in an infant. J Pediatr. 2011;159(5):869-869 e861.

5. Porzionato A, Macchi V, Rodriguez D, De Caro R. Airway obstruction by laryngeal masses in sudden and homicidal deaths. Forensic Sci Int. 2007;171(1):e15-20.

6. Walshe CM, Jonas N, Rohan D. Vallecular cyst causing a difficult intubation. Br J Anaesth. 2009;102(4):565.

7. Rivo J, Matot I. Asymptomatic vallecular cyst: airway management considerations. J Clin Anesth. 2001;13(5):383-386.

8. Vijayanand H, Mudhol RS, Patil PH, Maheswaran M. Innovative surgical management of large vallecular cysts. Indian J Otolaryngol Head Neck Surg. 2009;61(2):147149.

9. Abd Aziz A, Abdullah AF, Ahmad RA. Two cases of congenital vallecular cyst: a reminder of the potentially fatal cause of upper airway obstruction in infants. Malays J Med Sci. 2010;17(3):68-73.

10. Cheng KS, Ng JM, Li HY, Hartigan PM. Vallecular cyst and laryngomalacia in infants: report of six cases and airway management. Anesth Analg. 2002;95(5):12481250 , table of contents. 